\title{
Article \\ TET2 Inhibits PD-L1 Gene Expression in Breast Cancer Cells through Histone Deacetylation
}

\author{
Yinghui Shen ${ }^{1,+}$, Lu Liu ${ }^{1, \dagger}$, Mengyuan Wang ${ }^{1,+}{ }^{+}$Bo Xu ${ }^{1}$, Ruitu Lyu ${ }^{2}$, Yujiang Geno Shi ${ }^{3, *}$ and Li Tan ${ }^{1, * \mathbb{D}}$ \\ 1 Center for Medical Research and Innovation, Shanghai Pudong Hospital, Fudan University Pudong Medical \\ Center, and Shanghai Key Laboratory of Medical Epigenetics, Institutes of Biomedical Sciences, \\ Fudan University, Shanghai 200032, China; 16111510017@fudan.edu.cn (Y.S.);

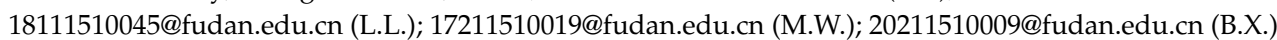 \\ 2 Department of Chemistry, University of Chicago, Chicago, IL 60637, USA; lvruitu@uchicago.edu \\ 3 Division of Endocrinology, Diabetes and Hypertension, Brigham and Women's Hospital, \\ Harvard Medical School, Boston, MA 02115, USA \\ * Correspondence: yujiang_shi@hms.harvard.edu (Y.G.S.); litan@fudan.edu.cn (L.T.); \\ Tel.: +86-21-54237876 (L.T.) \\ + These authors equally contributed to the study.
}

check for updates

Citation: Shen, Y.; Liu, L.; Wang, M.; Xu, B.; Lyu, R.; Shi, Y.G.; Tan, L. TET2 Inhibits $P D-L 1$ Gene Expression in Breast Cancer Cells through Histone Deacetylation. Cancers 2021, 13, 2207. https://doi.org/10.3390/cancers 13092207

Academic Editors: Lisardo Bosca,

Antonio Castrillo and

Eduardo Lopez-Collazo

Received: 13 April 2021

Accepted: 2 May 2021

Published: 4 May 2021

Publisher's Note: MDPI stays neutral with regard to jurisdictional claims in published maps and institutional affiliations.

Copyright: (c) 2021 by the authors. Licensee MDPI, Basel, Switzerland. This article is an open access article distributed under the terms and conditions of the Creative Commons Attribution (CC BY) license (https:/ / creativecommons.org/licenses/by/ $4.0 /)$.
Simple Summary: Programmed cell death ligand 1 (PD-L1) is an essential immune checkpoint molecule that helps tumor cells to escape the immune surveillance. The aim of the current study was to investigate the epigenetic mechanisms underlying the aberrant expression of PD-L1 in breast cancer cells. Here, we identified TET2 as a negative regulator of PD-L1 gene transcription in breast cancer cells. Mechanistically, TET2 recruits HDAC1/2 to the PD-L1 promoter and facilitates the deacetylation of $\mathrm{H} 3 \mathrm{~K} 27 \mathrm{ac}$, resulting to the suppression of PD-L1 gene transcription. Our work reveals an unanticipated role of TET2-HDAC1/2 complex in the regulation of PD-L1 gene expression, providing new insights into the epigenetic mechanisms that drive immune evasion during breast cancer pathogenesis.

Abstract: Activation of PD-1/PD-L1 checkpoint is a critical step for the immune evasion of malignant tumors including breast cancer. However, the epigenetic mechanism underlying the aberrant expression of PD-L1 in breast cancer cells remains poorly understood. To investigate the role of TET2 in the regulation of PD-L1 gene expression, quantitative reverse transcription PCR (RT-qPCR), Western blotting, chromatin immunoprecipitation (ChIP) assay and MeDIP/hMeDIP-qPCR were performed on MCF7 and MDA-MB-231 human breast cancer cells. Here, we reported that TET2 depletion upregulated PD-L1 gene expression in MCF7 cells. Conversely, ectopic expression of TET2 inhibited PD-L1 gene expression in MDA-MB-231 cells. Mechanistically, TET2 protein recruits histone deacetylases (HDACs) to PD-L1 gene promoter and orchestrates a repressive chromatin structure to suppress PD-L1 gene transcription, which is likely independent of DNA demethylation. Consistently, treatment with HDAC inhibitors upregulated PD-L1 gene expression in wild-type (WT) but not TET2 KO MCF7 cells. Furthermore, analysis of the CCLE and TCGA data showed a negative correlation between TET2 and PD-L1 expression in breast cancer. Taken together, our results identify a new epigenetic regulatory mechanism of PD-L1 gene transcription, linking the catalytic activity-independent role of TET2 to the anti-tumor immunity in breast cancer.

Keywords: TET2; PD-L1; HDAC; breast cancer; epigenetic regulation

\section{Introduction}

For the past few years, immunotherapy has emerged as a frontline treatment for multiple malignancies, and now joins the ranks of surgery, radiation, chemotherapy, and targeted therapy for cancer therapy [1]. Different from traditional therapies, cancer immunotherapy utilizes the body's own immune system to fight against tumor cells [2]. Among various 
immunotherapeutic strategies, the immune checkpoint blockade (ICB) has the broadest impact and prospects, with several antibodies targeting CTLA4 (cytotoxic T lymphocyte antigen 4), PD1 (programmed cell death 1), and PD-L1 (PD-1 ligand 1) approved by the FDA for the treatment of a number of different cancers [3-5]. PD-L1, encoded by the CD274 gene, is an essential immune checkpoint molecule that is mainly expressed on the surface of tumor cells and macrophages [6]. The expression of PD-L1 is commonly elevated in cancer cells $[7,8]$. Cancer cells may exhibit immune escape upon recognition of PD-L1 by PD-1, which mediates T cell exhaustion [9]. Therefore, understanding the regulatory mechanisms of $P D-L 1$ gene expression in cancer cells is of great importance for improving responsiveness to anti-PD-L1 immunotherapy and suppressing immune evasion.

Many studies have revealed the transcriptional regulatory mechanisms of the $P D$ L1 gene, including the inflammatory cytokines, specific transcription factors, and so on [? ]cers-1201219,B11-cancers-1201219,B12-cancers-1201219,B13-cancers-1201219. In addition, the epigenetic modifiers also play an important role in regulating PD-L1 gene transcription, altering the chromatin accessibility for the transcription factors through DNA methylation or histone modifications [14-17]. For instance, DNA methylation at the promoter region is commonly considered to be an epigenetic mechanism of transcriptional silencing [18]. In contrast, TET (Ten-eleven translocation) family members, including TET1, TET2, and TET3, have been regarded as DNA demethylases for gene activation $[19,20]$. TET proteins initiate active or passive DNA demethylation by promoting $5 \mathrm{mC}$ (5-methylcytosine) oxidization [21]. Recent studies have shown that TET2 could act as a critical player in the regulation of immune homeostasis and anti-tumor immunity [22-27]. Although a recent work reported that TET2 augments the IFN-gamma-induced PD-L1 expression in melanoma, colon cancer, and acute monocytic leukemia cells [15], whether TET2 is involved in the epigenetic regulation of $P D-L 1$ gene expression in breast cancer remains largely unknown.

In this study, we set out to investigate the relationship between TET2 and PD-L1 in breast cancer. Surprisingly, we found that TET2 is a suppressor of $P D-L 1$ gene transcription in breast cancer cells. Mechanistically, TET2 inhibits PD-L1 gene expression through recruiting HDAC1/2 to $P D$-L1 gene promoter and facilitating the histone deacetylation, which is likely independent of DNA methylation and hydroxymethylation.

\section{Materials and Methods}

\subsection{Cell Cultures}

MCF7 and MDA-MB-231 human breast cancer cells were cultured in a DMEM high glucose medium (Hyclone, Marlborough, MA, USA) supplemented with 10\% fetal bovine serum (FBS)(BI) and 1\% penicillin/streptomycin (100 U/mL, Hyclone). All cells were cultured at $37{ }^{\circ} \mathrm{C}$ in a humidified incubator containing $5 \% \mathrm{CO}_{2}$. The identity of two cell lines was confirmed by STR genotyping analysis.

\subsection{Stable Cell Lines Construction}

TET2 knockout MCF7 cells were generated by the CRISPR method as described previously [28]. Mono-cell colonies were picked and expanded for identification and future experiments. The TET2 knockout efficiency of these colonies was examined by Western blot analysis of TET2 protein expression and PCR analysis of genomic DNA for indels around the sgRNA targeting region. The following target sequences were used for sgRNA design:

TET2KO sg\#1: AGGACTCACACGACTATTC

TET2KO sg\#2: GGAGAAAGACGTAACTTCG

Mock (empty vector) and TET2-overexpressing (O/E) MDA-MB-231 cells were generated using the piggybac system as described previously [29]. In brief, MDA-MB-231 cells were co-transfected with pCMV-PBase and piggybac plasmids (pPB-CAG-ires-Pac empty vector or pPB-CAG-Flag-HA-TET2-ires-Pac). Puromycin $(2 \mu \mathrm{g} / \mathrm{mL})$ was added to the culture medium at $48 \mathrm{~h}$ post-transfection. The puromycin-resistant stable cell colonies were picked and expanded for identification and future experiments. 


\subsection{Reagents and Antibodies}

The following cytokines and chemical inhibitors were used for the treatment of cells in indicated experiments: IFN-gamma (Peprotech, Cranbury, NJ, USA, \#300-02), 5-Aza-CdR (5-aza-2'-deoxycytidine, Sigma-Aldrich, St. Louis, MO, USA, \#A3656), TSA (Trichostatin A, ApexBio, Houston, TX, USA, \#A8183), and SAHA (Vorinostat, Selleck, Houston, TX, USA, \#S1047).

The following antibodies were used for Western blot, ChIP, MeDIP and hMeDIP in our study: LaminB1 (Proteintech, \#66095-1-Ig), TET2 (CST, Boston, MA, USA, \#18950S), PD-L1 (CST, \#13684S), GAPDH (Abclonal, Wuhan, HB, China, \#AC033RRID), H3K27ac (Active Motif, Carlsbad, CA, USA, \#39133), H3K4me3 (CST, \#9751), H3K27me3 (CST, \#9733), HDAC1 (CST, \#34589S), HDAC2 (CST, \#57156S), 5hmC (Active Motif, \#39999), and 5mC (Active Motif, \#39649).

\subsection{Knocking Down by shRNAs}

The oligos for TET2 shRNAs were subcloned into the pLKO.1-TRC vector (Addgene, Watertown, MA, USA). The shRNA lentiviral particles were packaged in 293T cells according to the manufacturer's guidelines. MCF7 cells were infected with each lentivirus supernatant in the presence of $8 \mu \mathrm{g} / \mathrm{mL}$ polybrene. At $48 \mathrm{~h}$ post infection, the infected cells were cultured with $2 \mu \mathrm{g} / \mathrm{mL}$ puromycin. After continuous puromycin screening for 5 days, the survival MCF7 cells were collected for RNA isolation and RNA-seq.

\section{5. $R T-q P C R$}

RNA was extracted from the cells using TRIzol reagent (Thermo Fisher Scientific, Waltham, MA, USA) according to the manufacturer's protocol. The protocol of RT-qPCR was described in our previous study [13]. Gene expression levels were normalized to GAPDH. The sequences of primers were shown in Supplementary Table S4.

\subsection{Western Blot}

Cells were harvested and lysed with 1x SDS loading buffer. After quantification of the lysates with BCA assay, the Western blot assay was performed as described previously [13]. The primary antibodies used in this study were TET2 (1:1000), PD-L1 (1:1000), laminB1 (1:5000), H3K27ac (1:2000), and GAPDH (1:10,000).

\section{7. $C h I P-q P C R$}

The Chromatin immunoprecipitation (ChIP) assay was performed as previously described [29]. The enrichment levels of TET2, H3K4me3, H3K27me3, H3K27ac, and HDAC1/2 at $P D-L 1$ promoter were quantified by qPCR analysis of the ChIP products and the relevant inputs. Primer sequences used for ChIP-qPCR are listed in Supplementary Table S4.

\subsection{MeDIP/hMeDIP-qPCR}

Genome DNA was prepared from cells using DNeasy Blood \& Tissue kit (QIAGEN). $\mathrm{MeDIP} / \mathrm{hMeDIP}$-qPCR was performed as described previously [30]. Enrichment of 5mC (5-methylcytosine) or $5 \mathrm{hmC}$ (5-hydroxymethylcytosine) at $P D-L 1$ promoter was quantified by qPCR analysis. Primer sequences used for MeDIP/hMeDIP-qPCR are listed in Supplementary Table S4.

\subsection{Co-Immunoprecipitation}

The co-immunoprecipitation assay was conducted as previously described [31].

\subsection{RNA seq Analysis}

RNA samples from MCF7 cells (WT, TET2 KO \#1, TET2 KO\#2, scramble, shTET2\#1, and shTET2\#2) were subjected for RNA-seq using the Illumina platform. First, raw reads were trimmed to remove adapters and low-quality bases using the trim_galore program (version 0.6.6) with parameters: "-paired-fastqc". Secondly, trimmed fastq files were 
aligned to the human reference genome (hg19.fa from UCSC) with the tophat program (v2.1.1) with default parameters. We used the FPKM_count.py (RSeQC) to calculate FPKM (fragments per kilo base of transcript per $\mathrm{M}$ ) to represent the abundance of gene expression. The bedgraph files were uploaded on UCSC genome browser for visualization. RNA-seq data has been deposited in the NCBI Gene Expression Omnibus (GEO) under the accession number GSE164032 (https: / / www.ncbi.nlm.nih.gov / geo / query / acc.cgi?acc=GSE164032, Submitted on 30 December 2020, Released on 1 June 2021).

\subsection{Analysis of CCLE and TCGA Data}

The gene expression data of 23 kinds of cancer cell lines were downloaded from CCLE (Comprehensive Cell Line Encyclopedia, http:/ / www.broadinstitute.org/ccle/home; accessed on 2 May 2018). The expression levels of TET2 and PD-L1 from breast invasive carcinoma (Agilent microarray data) were downloaded from TCGA (The Cancer Genome Atlas, http:/ / www.cbioportal.org; accessed on 19 March 2020). The TET2 and PD-L1 mRNA levels of cancer cell lines and breast cancer tissues were used for correlation analysis and linear regression analysis.

\subsection{Statistical Analysis}

GraphPad Prism Software was used for quantitative data visualization and statistical analysis. All graphs were presented as an average of at least three independent experiments. Standard deviation (S.D.) was calculated and presented as error bars in graphs. Comparisons between two groups were analyzed by paired Student's $t$-test. Multiple comparisons were analyzed by one-way ANOVA with Tukey post-test. Significance level was set as $p=0.05$ and presented as ${ }^{*}$ in all graphs $\left({ }^{*}, p<0.05 ;{ }^{* *}, p<0.01 ; * * *, p<0.001 ; * * * *, p<0.0001\right.$; ns, not significant).

\section{Results}

\subsection{TET2 Is a Negative Regulator of PD-L1 Gene Transcription in Breast Cancer Cells}

In an RNA-seq analysis for the downstream genes of TET2 in MCF7 cells, we identified that PD-L1 mRNA expression level was upregulated in TET2 KO MCF7 cells compared to wild type (WT) MCF7 cells (Figure 1A). RT-qPCR analysis also confirmed the substantial increase in PD-L1 mRNA levels in TET2 KO MCF7 cells (Figure 1B). Given that the basal expression level of $P D-L 1$ is relatively low in MCF7 cells, we also treated cells with IFNgamma and measured the impact of TET2 depletion on PD-L1 expression. RT-qPCR and Western blotting analyses showed that IFN-gamma treatment dramatically upregulated $P D$ L1 expression in both WT and TET2 KO MCF7 cells (Figure 1C,D). Importantly, regardless of the absence or presence of IFN-gamma stimulation, the mRNA and protein levels of $P D-L 1$ in TET2 KO MCF7cells were correspondingly higher than those in WT MCF7 cells (Figure $1 \mathrm{C}, \mathrm{D}$ ). Furthermore, $P D-L 1$ intron mRNA level displayed a similar increase in TET2 KO MCF7 cells (Figure S1A), indicating a direct effect of TET2 depletion on nascent RNA synthesis rather than mRNA stability. Knocking down TET2 by shRNA also increased the PD-L1 mRNA level in MCF7 cells (Figure S1B). Moreover, the MDA-MB-231 cell is a commonly used triple-negative breast cancer cell line in laboratory research. Compared to MCF7 cells, MDA-MB-231 cells express relatively low levels of TET2 expression but high levels of PD-L1 expression, showing features of an ideal model for a TET2 "gain-of-function" study. To verify the suppressive role of TET2 on PD-L1 expression, we overexpressed TET2 in MDA-MB-231 cells and observed the downregulation of $P D-L 1$ gene expression upon TET2 overexpression (Figure S2A,B). Overall, our data demonstrate that TET2 functions as a negative regulator of $P D-L 1$ gene transcription in breast cancer cells. 
A

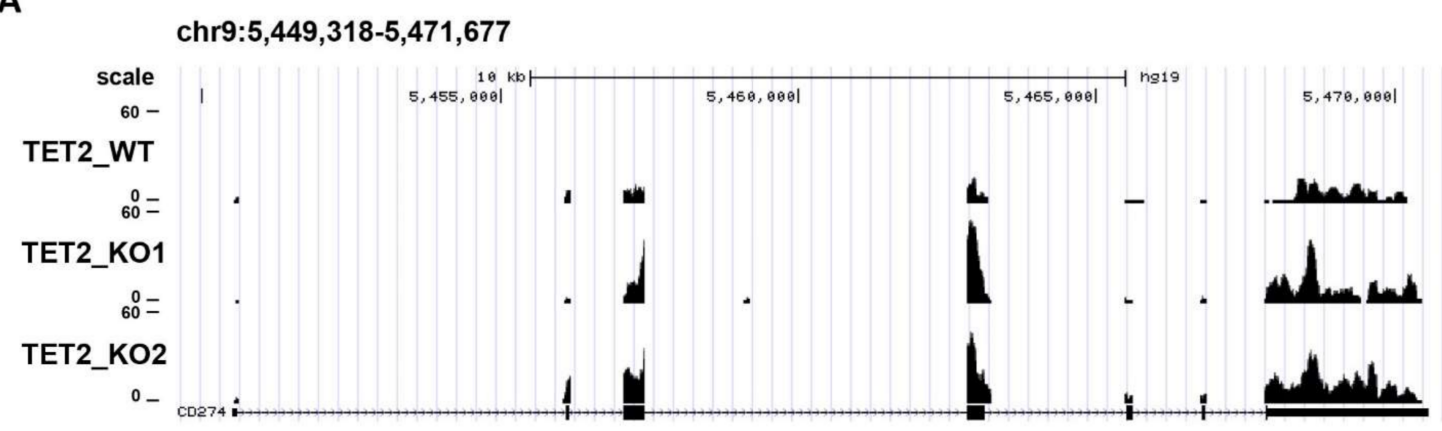

B

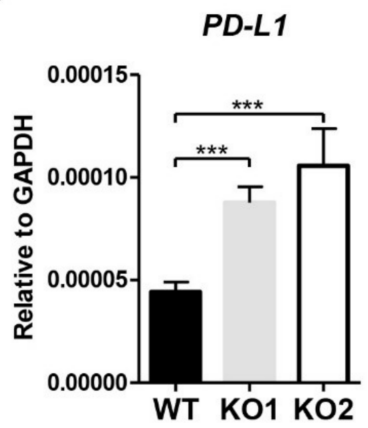

C

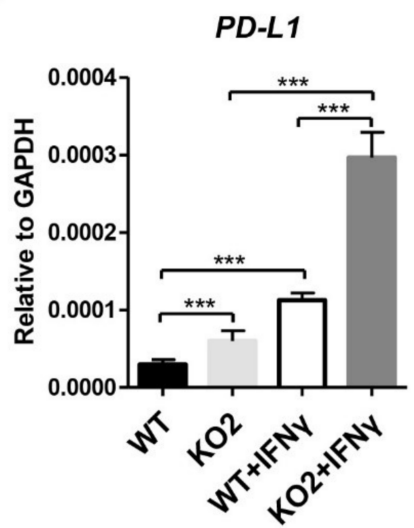

D

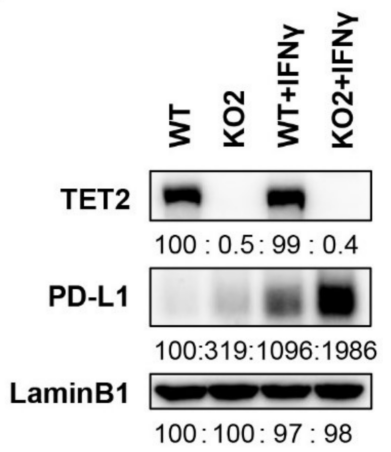

Figure 1. TET2 is a negative regulator of $P D-L 1$ gene transcription in breast cancer cells. (A) RNA-seq snapshot of $P D$ - $L 1$ (CD274) gene expression in WT, TET2_KO1 and TET2_KO2 MCF7 cells. (B) RT-qPCR analysis of the relative mRNA expression levels of PD-L1 in WT, KO1, and KO2 MCF7 cells. (C) RT-qPCR analysis of the relative mRNA expression levels of PD-L1 in WT and TET2 KO MCF7 cells with or without IFN-gamma $(100 \mathrm{ng} / \mathrm{mL})$ stimulation. (D) Western blot analysis of the PD-L1 protein levels in WT and TET2 KO MCF7 cells with or without IFN-gamma (100 ng/mL) stimulation. $(* * *, p<0.001$.)

\subsection{TET2 KO Does Not Alter the DNA Methylation and Hydroxymethylation Level at the Promoter Region of PD-L1 Gene}

Next, we investigate the molecular mechanism through which TET2 inhibits $P D$ L1 gene transcription in breast cancer cells. By analyzing the published TET2 ChIP-seq data (GSE120756) [32], we discovered that TET2 directly bound to the promoter region of PD-L1 gene in MCF7 cells (Figure 2A). Our TET2 ChIP-qPCR data also confirmed the occupancy of TET2 at the PD-L1 promoter, indicating a direct action of TET2 on PD-L1 gene transcription (Figure $2 \mathrm{~B}$ ). Since TET2 has the ability to catalyze $5 \mathrm{mC}$ oxidation and initiate DNA demethylation, we examined the $5 \mathrm{mC}$ and $5 \mathrm{hmC}$ enrichment at the promoter region of PD-L1 gene by MeDIP- and hMeDIP-qPCR. Unexpectedly, TET2 depletion has no significant effect on the $5 \mathrm{mC}$ and $5 \mathrm{hmC}$ enrichment at $P D-L 1$ promoter region (Figure $2 \mathrm{C}, \mathrm{D}$ ). Given the critical role of TET2 in DNA demethylation, TET2 KO may increase DNA methylation levels on other genomic regulatory regions beyond the $P D-L 1$ promoter, which might contribute to the transcriptional activation of $P D-L 1$ gene. To exclude this possibility, we treated WT and TET2 KO MCF7 cells with 5-Aza-CdR (a DNMT inhibitor) and examined the mRNA expression of the PD-L1 gene. Figure 2E showed that 5-Aza-CdR treatment was not sufficient to change PD-L1 expression in either WT or TET2 KO MCF-7 cells. Thus, our data suggest that TET2-mediated inhibition of PD-L1 gene transcription is not dependent on its $5 \mathrm{mC}$ dioxygenase activity. 
A

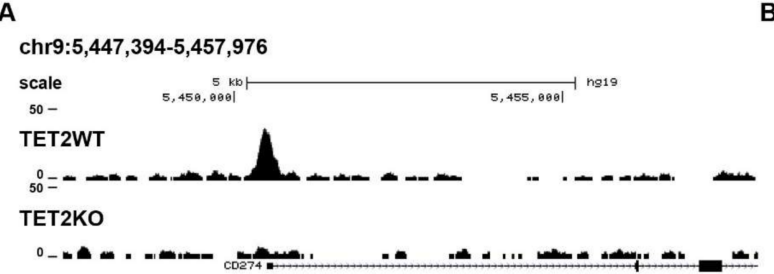

B

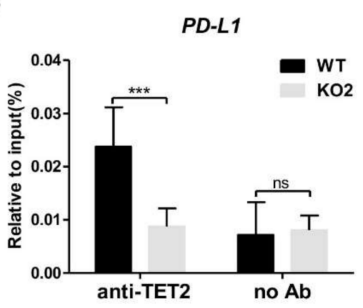

C

D
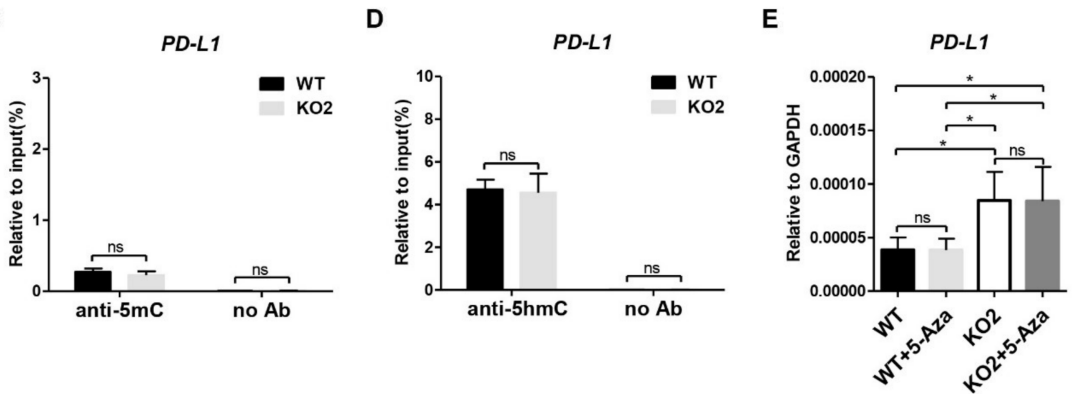

Figure 2. TET2 KO does not alter the DNA methylation and hydroxymethylation level at the promoter region of PD-L1 gene. (A) Snapshot of TET2 ChIP-seq data (GSE120756) at PD-L1 (CD274) promoter in WT and TET2 KO MCF7 cells. (B) ChIP-qPCR validation of TET2 occupancy at PD-L1 promoter in WT and TET2 KO MCF7 cells. (C) MeDIP-qPCR analysis of $5 \mathrm{mC}$ enrichment at PD-L1 promoter in WT and TET2 KO MCF7 cells. (D) hMeDIP-qPCR analysis of $5 \mathrm{hmC}$ enrichment at PD-L1 promoter in WT and TET2 KO MCF7 cells. (E) RT-qPCR analysis of the relative mRNA expression levels of PD-L1 in WT and TET2 KO MCF7 cells with or without $10 \mu \mathrm{M}$ 5-Aza treatment. $\left({ }^{*}, p<0.05 ;{ }^{* * *}, p<0.001\right.$; ns, not significant.)

\subsection{TET2 Recruits HDAC1/2 to Deacetylate H3K27ac at PD-L1 Promoter}

In addition to the DNA-demethylating activity, TET2 can also function as a transcriptional co-factor to regulate gene expression [33]. We profiled the well-studied histone modifications (H3K4me3, H3K27me3, and H3K27ac) at the PD-L1 promoter region in WT and TET2 KO MCF7 cells. ChIP assay showed that the levels of H3K4me3 enrichment at PD-L1 promoter were comparable between WT and TET2 KO MCF7 cells (Figure 3A), while the repressive H3K27me3 was rare at the PD-L1 promoter in WT and TET2 KO MCF7 cells (Figure $3 \mathrm{~B}$ ). Consistent to the changes of $P D-L 1$ gene transcription, the H3K27ac levels at PD-L1 promoter region were increased in MCF7 cells upon TET2 depletion (Figure 3C). Additionally, TET2 overexpression reduced H3K27ac enrichment at the PD-L1 promoter region in MDA-MB-231 cells (Figure S3A). Our data suggest that TET2 is able to modulate the H3K27ac level at the PD-L1 promoter in breast cancer cells.

TET2 has been shown to recruit histone deacetylases (HDAC1/2) to specific gene loci, thereby mediating the transcriptional repression in immune cells $[34,35]$. Therefore, we performed ChIP-qPCR analysis of HDAC1 and HDAC2 in WT and TET2 KO MCF7 cells. As expected, the binding of HDAC1 and HDAC2 to the PD-L1 promoter in TET2 KO MCF7 cells was significantly lower than that in WT cells (Figure 3D,E). Moreover, we conducted a co-IP assay for the interaction between TET2 and HDAC1/2 using anti-HDAC specific antibody. As expected, we detected a TET2 band in the anti-HDAC1 immunoprecipitants from MCF7 cells and TET2-O/E MDA-MB-231 cells by Western blotting (Figure 3F \& Figure S3C). Next, we chose two HDAC inhibitors (TSA and SAHA) to treat WT and TET2 KO MCF7 cells. As shown in Figure 3G, both inhibitors increased the global H3K27ac level in WT and TET2 KO MCF7 cells. Importantly, the PD-L1 mRNA expression level was elevated by treatment with HDAC inhibitors only in WT cells but not in TET2 KO MCF7 cells (Figure $3 \mathrm{H}$ ). We also observed that HDAC1 binding at the PD-L1 gene promoter was increased in TET2 O/E MDA-MB-231 cells (Figure S3B). Taken together, our data suggest a working model in which TET2 unites HDAC1/2 to deacetylate H3K27ac and establishes a repressive chromatin structure at the $P D-L 1$ promoter (Figure $3 \mathrm{I}$ ). 
A
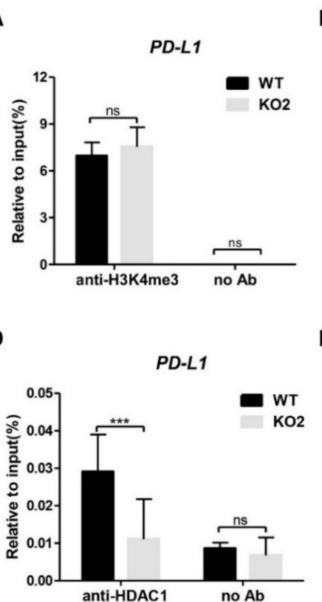

B

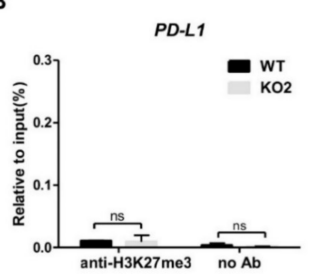

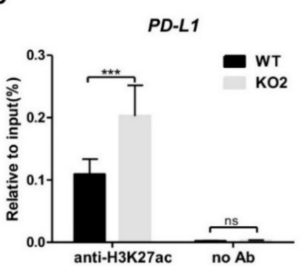

F

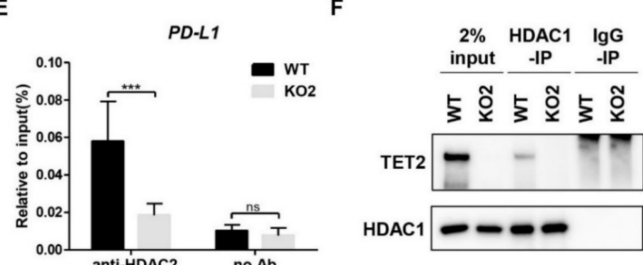

G

H
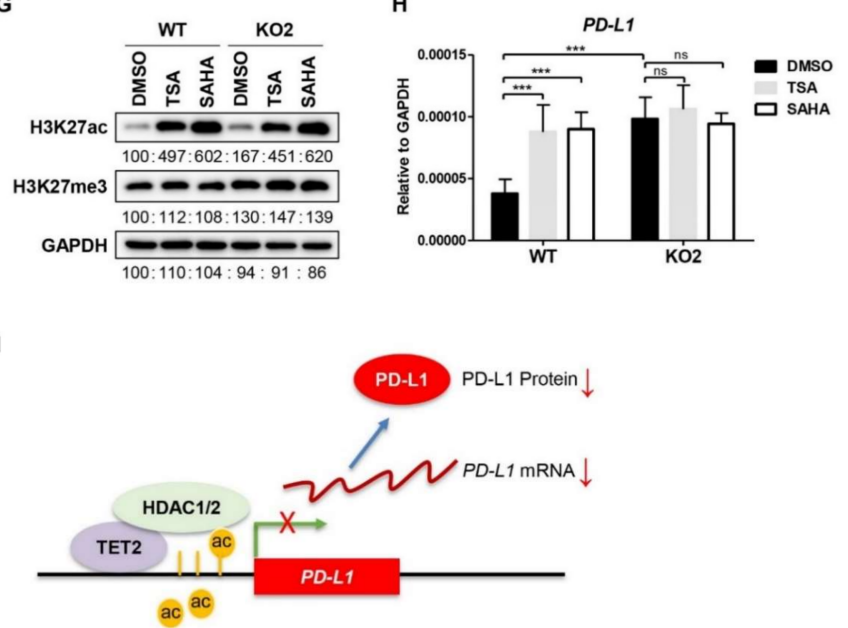

Figure 3. TET2 recruits HDAC1/2 to deacetylate H3K27ac at $P D-L 1$ promoter. (A) ChIP-qPCR analysis of H3K4me3 enrichment at PD-L1 promoter in WT and TET2 KO MCF7 cells. (B) ChIPqPCR analysis of H3K27me3 enrichment at PD-L1 promoter in WT and TET2 KO MCF7 cells. (C) ChIP-qPCR analysis of H3K27ac enrichment at PD-L1 promoter in WT and TET2 KO MCF7 cells. (D,E) ChIP-qPCR analysis of the occupancy of HDAC1 and HDAC2 at the PD-L1 promoter in WT and TET2 KO MCF7 cells. (F) Western blot analysis of the anti-HDAC1-IP and IgG-IP products in WT and TET2 KO MCF7 cells. (G) Western blot analysis of the global H3K27ac levels in WT and TET2 KO MCF7 cells treated with or without HDAC inhibitors (TSA $1 \mu \mathrm{M}$; SAHA $5 \mu \mathrm{M}$ ). (H) RT-qPCR analysis of the relative mRNA expression levels of PD-L1 in WT and TET2 KO MCF7 cells treated with or without HDAC inhibitors (TSA $1 \mu \mathrm{M}$; SAHA $5 \mu \mathrm{M}$ ). (I) Schematic diagram of the working model in which TET2 inhibits PD-L1 gene transcription through HDAC1/2-mediated histone deacetylation. (***, $p<0.001$; ns, not significant.)

\subsection{Negative Correlation between TET2 and PD-L1 Expression Levels in Breast Cancer}

To investigate the clinical significance of the TET2/PD-L1 axis, we analyzed the relationship between TET2 and PD-L1 expression levels in online breast cancer data. CCLE data analysis showed that TET2 mRNA expression levels are negatively associated with PD-L1 mRNA expression levels in 57 breast cancer cell lines $(p=0.0031)$ (Figure 4A). Interestingly, by analyzing other types of cancer cell lines from CCLE, we found that at least two other kinds of cancer types (lung and soft tissue) had a significant negative correlation between TET2 and PD-L1 mRNA expression levels (Table S1). We also analyzed the correlation between TET2 and PD-L1 by separating the CCLE breast cancer cell lines into three tumor subtypes and found that TET2 expression is negatively correlated with PD-L1 expression in luminal subtype $(p=0.0282)$ (Table S2). In a similar manner, TCGA data analysis also 
showed that TET2 expression levels had a negative correlation with PD-L1 expression levels in breast cancer $(n=1904, p=0.0065)$ (Figure 4B). Through an analysis of the four subtypes of TCGA breast cancer data, we found that TET2 and PD-L1 showed a negative correlation only in luminal B and Her2-enriched subtypes (Table S3).

A

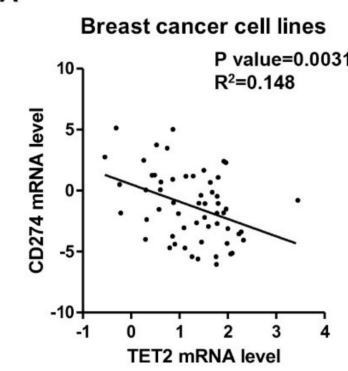

B

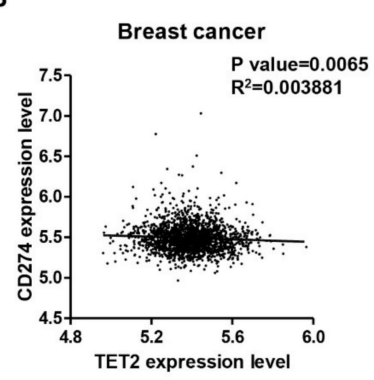

Figure 4. A negative correlation between TET2 and $P D-L 1$ gene transcription in breast cancer. (A) CCLE data analysis showing a significant negative correlation between TET2 and PD-L1 expression levels in breast cancer cell lines $(n=57)$. (B) TCGA data analysis showing a mild but significant negative correlation between TET2 and PD-L1 expression levels in breast cancer tissues $(n=1904)$.

\section{Discussion}

In this study, we demonstrate that TET2 inhibits $P D-L 1$ gene expression in breast cancer cells either under baseline conditions or upon IFN-gamma stimulation. Conversely, a recent work from $\mathrm{Xu}$ et al. [15] showed that IFN-gamma-induced PD-L1 gene expression was impaired by TET2 depletion in murine melanoma (B16-OVA), colon tumor cells (MC38), and human monocytic cells (THP-1). Mechanistically, they revealed that TET2 could be recruited by STAT1 to hydroxymethylate the $P D-L 1$ gene promoter and enhance its transcription in murine melanoma and colon tumor upon IFN-gamma stimulation. However, our data showed that the PD-L1 promoter of breast cancer cells is in a DNA hypo-methylated status and that TET2 depletion does not alter the DNA methylation or hydroxymethylation levels at the promoter region of $P D-L 1$ gene. Moreover, we found that treatment with 5-Aza-CdR, a DNMT inhibitor, could not enhance $P D-L 1$ gene expression in either WT or TET2 KO MCF7 cells, suggesting that TET2 may repress PD-L1 gene expression in a catalytic-activity independent manner. These opposite results indicate that TET2-mediated regulation of PD-L1 gene expression may be largely dependent on the cancer/tissue types.

In addition to the well-known DNA demethylation activity, TET proteins have been shown to recruit histone deacetylases (HDACs) and mediate transcriptional repression in immune cells [34-36]. Coincidentally, our study demonstrates that TET2 recruits HDAC1/2 to deacetylate $\mathrm{H} 3 \mathrm{~K} 27 \mathrm{ac}$ at $P D-L 1$ promoter and results in the transcriptional suppression of $P D-L 1$ gene. Although our work identifies that TET2 acts as a scaffold protein for the negative regulation of $P D-L 1$ gene transcription in breast cancer cells, it is still unclear how TET2 itself is recruited to the $P D-L 1$ promoter. Since dozens of transcription factors have been identified to recruit TET2 to specific gene loci for epigenetic regulation in different types of tissues [37-39], we speculate that a specific transcription factor may be responsible for this task in breast cancer.

Previous reports have showed that the downregulation of TET2 expression and $5 \mathrm{hmC}$ levels is an epigenetic hallmark of multiple types of cancers [21,40-42]. Dysregulation of the TET2/5hmC pathway promotes epithelial-mesenchymal transition (EMT), chemotherapy resistance, proliferation, invasion, and metastasis during breast cancer pathogenesis [43-45]. Based on the afore-mentioned regulatory mechanism, we speculate that TET2 loss may facilitate immune evasion by breast cancer cells through upregulating PD-L1 gene expression. Consistently, we observed an inverse correlation between PD-L1 and TET2 expression levels regardless of whether the breast cancer cell lines were cultured in vitro (CCLE data) or in cancer samples from patients (TCGA data). Therefore, our work expands 
our current understanding of the pleiotropic role of TET2 loss in breast cancer pathogenesis, especially as it relates to immune evasion.

Interestingly, by analyzing multiple cancer cell lines from CCLE, we noticed that the correlation between TET2 and PD-L1 in lung cancer cell lines is also negative and more significant than that in breast cancer cell lines. This finding suggests that the TET2/PDL1 negative regulatory axis may exist not only in breast cancer but also in other types of cancers. Given that anti-PD-1/L1 therapy has been successfully applied in the firstline therapy of lung cancer, it is of great interest to validate this observation in clinical patient samples and explore the prognostic value of TET2 expression in predicting the responsiveness of lung cancer patients to anti-PD-1/L1 therapy. If true, it may provide valuable clues and new strategies to improve immunotherapy treatment of lung cancer.

Multiple signaling pathways are aberrantly activated in the complicated tumor microenvironment of breast cancers. Among them, several signaling pathways (such as hypoxia, AMPK, IFN, and oxidative stress) have been reported to regulate TET2 expression level. The findings of our study suggest that the tumor microenvironment may modulate the expression of PD-L1 gene in breast cancer through targeting the TET2/HDAC complex. Given that anti-PD-1/L1 immunotherapy has been widely used in clinics, we speculate that the combination of HDAC inhibitors and targeting TET2 with anti-PD-L1 immunotherapy may be a new strategy for breast cancer patients who have low responsiveness to anti-PD-1/L1 immunotherapy. Currently, four HDAC inhibitors, Vorinostat, Romidepsin, Belinostat, and Panobinostat have been approved by FDA for cancer treatment [46]. Several studies have reported that HDAC inhibition make for increased PD-L1 expression in melanoma [47], ARID1A-inactivated Ovarian Cancer [48], and anaplastic thyroid cancer [49]. These findings, together with ours, reinforce a rationale for applying HDAC inhibitors or targeting TET2 to augment the immunotherapy of breast cancer.

\section{Conclusions}

In conclusion, our study provides clear evidences that $P D-L 1$ gene transcription is negatively regulated by the TET2-HDAC1/2 complex in breast cancer cells. Although more work remains to be done with regard to the regulatory mechanism and functional role of TET2/PD-L1 axis in the anti-tumor immunity, our findings suggest that targeting TET2 or HDAC1/2 might be a potential combination strategy for the anti-PD-1/PD-L1 immunotherapy treatment of breast cancer.

Supplementary Materials: The following are available online at https:/ / www.mdpi.com/article/10 .3390/cancers13092207/s1, Figure S1: PD-L1 is negatively regulated by TET2 in MCF7 cells; Figure S2: TET2 overexpression inhibits PD-L1 expression in MDA-MB-231 cells. Figure S3: TET2 is required for H3K27 deacetylation and HDAC occupancy at PD-L1 promoter in MDA-MB-231; Table S1 \& S2: CCLE data analysis; Table S3: TCGA data analysis; Table S4: Primers used in the study; Figure S4: Original Western blots figures.

Author Contributions: Conceptualization, L.T. and Y.G.S.; Acquisition of data, Y.S., L.L., and M.W.; Validation, B.X.; Bioinformatics analysis, R.L.; Writing-original draft preparation, Y.S., L.L., M.W., Y.G.S., and L.T.; Writing - review and editing, all authors; Project administration, L.T.; Funding Acquisition, L.T. All authors have read and agreed to the published version of the manuscript.

Funding: This work was supported by the National Natural Science Foundation of China (81672785, 31871291, and 82073113 to L.T.) and the National Key R\&D Project of China (2016YFA0101800 to L.T.). L.T. was also supported by the innovative research team of high-level local university in Shanghai.

Institutional Review Board Statement: Not applicable for no studies involving humans or animals. Informed Consent Statement: Not applicable for no studies involving humans.

Data Availability Statement: The RNA-seq data presented in this study are openly available under the accession number GSE164032 (https: / / www.ncbi.nlm.nih.gov/geo/query/acc.cgi?acc=GSE164 032), Submitted on 30 December 2020, Released on 1 June 2021. 
Acknowledgments: The authors would like to thank Lingchun Kong for constructing the TET2 O/E MDA-MB-231 cells and Zhennan Shi for constructing the TET2 KO MCF7 cells.

Conflicts of Interest: The authors declare no conflict of interest.

\section{References}

1. Sharma, P.; Allison, J.P. The future of immune checkpoint therapy. Science 2015, 348, 56-61. [CrossRef]

2. Farkona, S.; Diamandis, E.P.; Blasutig, I.M. Cancer immunotherapy: The beginning of the end of cancer? BMC Med. 2016, 14, 73. [CrossRef] [PubMed]

3. Gubin, M.M.; Zhang, X.; Schuster, H.; Caron, E.; Ward, J.P.; Noguchi, T.; Ivanova, Y.; Hundal, J.; Arthur, C.D.; Krebber, W.J.; et al. Checkpoint blockade cancer immunotherapy targets tumour-specific mutant antigens. Nature 2014, 515, 577-581. [CrossRef] [PubMed]

4. Ribas, A.; Wolchok, J.D. Cancer immunotherapy using checkpoint blockade. Science 2018, 359, 1350-1355. [CrossRef]

5. Wu, X.; Gu, Z.; Chen, Y.; Chen, B.; Chen, W.; Weng, L.; Liu, X. Application of PD-1 Blockade in Cancer Immunotherapy. Comput. Struct. Biotechnol. J. 2019, 17, 661-674. [CrossRef]

6. Akinleye, A.; Rasool, Z. Immune checkpoint inhibitors of PD-L1 as cancer therapeutics. J. Hematol. Oncol. 2019, 12, 92. [CrossRef] [PubMed]

7. Green, M.R.; Monti, S.; Rodig, S.J.; Juszczynski, P.; Currie, T.; O'donnell, E.; Chapuy, B.; Takeyama, K.; Neuberg, D.; Golub, T.R.; et al. Integrative analysis reveals selective 9p24.1 amplification, increased PD-1 ligand expression, and further induction via JAK2 in nodular sclerosing Hodgkin lymphoma and primary mediastinal large B-cell lymphoma. Blood 2010, 116, 3268-3277. [CrossRef]

8. Kataoka, K.; Shiraishi, Y.; Takeda, Y.; Sakata, S.; Matsumoto, M.; Nagano, S.; Maeda, T.; Nagata, Y.; Kitanaka, A.; Mizuno, S.; et al. Aberrant PD-L1 expression through 3'-UTR disruption in multiple cancers. Nature 2016, 534, 402-406. [CrossRef]

9. Chen, J.; Jiang, C.C.; Jin, L.; Zhang, X.D. Regulation of PD-L1: A novel role of pro-survival signalling in cancer. Ann. Oncol. 2016, 27, 409-416. [CrossRef]

10. Sun, C.; Mezzadra, R.; Schumacher, T.N. Regulation and Function of the PD-L1 Checkpoint. Immunity 2018, 48, 434-452. [CrossRef]

11. Zerdes, I.; Matikas, A.; Bergh, J.; Rassidakis, G.Z.; Foukakis, T. Genetic, transcriptional and post-translational regulation of the programmed death protein ligand 1 in cancer: Biology and clinical correlations. Oncogene 2018, 37, 4639-4661. [CrossRef]

12. Vranic, S.; Cyprian, F.S.; Gatalica, Z.; Palazzo, J. PD-L1 status in breast cancer: Current view and perspectives. In Seminars in Cancer Biology; Academic Press: Cambridge, MA, USA, 2019.

13. Liu, L.; Shen, Y.; Zhu, X.; Lv, R.; Li, S.; Zhang, Z.; Shi, Y.G.; Tan, L. ERalpha is a negative regulator of PD-L1 gene transcription in breast cancer. Biochem. Biophys. Res. Commun. 2018, 505, 157-161. [CrossRef]

14. Yang, H.; Bueso-Ramos, C.; Dinardo, C.; Estecio, M.R.; Davanlou, M.; Geng, Q.R.; Fang, Z.; Nguyen, M.; Pierce, S.; Wei, Y.; et al. Expression of PD-L1, PD-L2, PD-1 and CTLA4 in myelodysplastic syndromes is enhanced by treatment with hypomethylating agents. Leukemia 2014, 28, 1280-1288. [CrossRef]

15. Xu, Y.P.; Lv, L.; Liu, Y.; Smith, M.D.; Li, W.C.; Tan, X.M.; Cheng, M.; Li, Z.; Bovino, M.; Aube, J.; et al. Tumor suppressor TET2 promotes cancer immunity and immunotherapy efficacy. J. Clin. Investig. 2019, 129, 4316-4331. [CrossRef]

16. Sasidharan Nair, V.; El Salhat, H.; Taha, R.Z.; John, A.; Ali, B.R.; Elkord, E. DNA methylation and repressive H3K9 and H3K27 trimethylation in the promoter regions of PD-1, CTLA-4, TIM-3, LAG-3, TIGIT, and PD-L1 genes in human primary breast cancer. Clin. Epigenet. 2018, 10, 78. [CrossRef]

17. Kumar, S.; Sharawat, S.K. Epigenetic regulators of programmed death-ligand 1 expression in human cancers. Transl. Res. 2018, 202, 129-145. [CrossRef]

18. Schubeler, D. Function and information content of DNA methylation. Nature 2015, 517, 321-326. [CrossRef]

19. Hu, L.; Lu, J.; Cheng, J.; Rao, Q.; Li, Z.; Hou, H.; Lou, Z.; Zhang, L.; Li, W.; Gong, W.; et al. Structural insight into substrate preference for TET-mediated oxidation. Nature 2015, 527, 118-122. [CrossRef]

20. Tahiliani, M.; Koh, K.P.; Shen, Y.; Pastor, W.A.; Bandukwala, H.; Brudno, Y.; Agarwal, S.; Iyer, L.M.; Liu, D.R.; Aravind, L.; et al. Conversion of 5-methylcytosine to 5-hydroxymethylcytosine in mammalian DNA by MLL partner TET1. Science 2009, 324, 930-935. [CrossRef]

21. Tan, L.; Shi, Y.G. Tet family proteins and 5-hydroxymethylcytosine in development and disease. Development 2012, 139, 1895-1902. [CrossRef]

22. Zhang, Q.; Casanova, J.L. Human TET2 bridges cancer and immunity. Blood 2020, 136, 1018-1019. [CrossRef]

23. Rosikiewicz, W.; Chen, X.; Dominguez, P.M.; Ghamlouch, H.; Aoufouchi, S.; Bernard, O.A.; Melnick, A.; Li, S. TET2 deficiency reprograms the germinal center B cell epigenome and silences genes linked to lymphomagenesis. Sci. Adv. 2020, 6, eaay5872. [CrossRef]

24. Pan, W.; Zhu, S.; Qu, K.; Meeth, K.; Cheng, J.; He, K.; Ma, H.; Liao, Y.; Wen, X.; Roden, C.; et al. The DNA Methylcytosine Dioxygenase Tet2 Sustains Immunosuppressive Function of Tumor-Infiltrating Myeloid Cells to Promote Melanoma Progression. Immunity 2017, 47, 284-297.e5. [CrossRef]

25. Cong, B.; Zhang, Q.; Cao, X. The function and regulation of TET2 in innate immunity and inflammation. Protein Cell 2020, 1-9. [CrossRef] 
26. Li, S.; Feng, J.; Wu, F.; Cai, J.; Zhang, X.; Wang, H.; Fetahu, I.S.; Iwanicki, I.; Ma, D.; Hu, T.; et al. TET2 promotes anti-tumor immunity by governing G-MDSCs and CD8+ T-cell numbers. EMBO Rep. 2020, 21, e49425. [CrossRef]

27. Fraietta, J.A.; Nobles, C.L.; Sammons, M.A.; Lundh, S.; Carty, S.A.; Reich, T.J.; Cogdill, A.P.; Morrissette, J.J.D.; Denizio, J.E.; Reddy, S.; et al. Disruption of TET2 promotes the therapeutic efficacy of CD19-targeted T cells. Nature 2018, 558, 307-312. [CrossRef]

28. Ran, F.A.; Hsu, P.D.; Wright, J.; Agarwala, V.; Scott, D.A.; Zhang, F. Genome engineering using the CRISPR-Cas9 system. Nat. Protoc. 2013, 8, 2281-2308. [CrossRef]

29. Kong, L.; Tan, L.; Lv, R.; Shi, Z.; Xiong, L.; Wu, F.; Rabidou, K.; Smith, M.; He, C.; Zhang, L.; et al. A primary role of TET proteins in establishment and maintenance ofDe Novobivalency at CpG islands. Nucleic Acids Res. 2016, 44, 8682-8692. [CrossRef]

30. Tan, L.; Xiong, L.; Xu, W.; Wu, F.; Huang, N.; Xu, Y.; Kong, L.; Zheng, L.; Schwartz, L.; Shi, Y.; et al. Genome-wide comparison of DNA hydroxymethylation in mouse embryonic stem cells and neural progenitor cells by a new comparative hMeDIP-seq method. Nucleic Acids Res. 2013, 41, e84. [CrossRef]

31. Xu, W.; Li, J.; He, C.; Wen, J.; Ma, H.; Rong, B.; Diao, J.; Wang, L.; Wang, J.; Wu, F.; et al. METTL3 regulates heterochromatin in mouse embryonic stem cells. Nature 2021, 591, 317-321. [CrossRef]

32. Wang, L.; Ozark, P.A.; Smith, E.R.; Zhao, Z.; Marshall, S.A.; Rendleman, E.J.; Piunti, A.; Ryan, C.; Whelan, A.L.; Helmin, K.A.; et al. TET2 coactivates gene expression through demethylation of enhancers. Sci. Adv. 2018, 4, eaau6986. [CrossRef] [PubMed]

33. Chen, Q.; Chen, Y.; Bian, C.; Fujiki, R.; Yu, X. TET2 promotes histone O-GlcNAcylation during gene transcription. Nature 2013, 493, 561-564. [CrossRef] [PubMed]

34. Zhang, Q.; Zhao, K.; Shen, Q.; Han, Y.; Gu, Y.; Li, X.; Zhao, D.; Liu, Y.; Wang, C.; Zhang, X.; et al. Tet2 is required to resolve inflammation by recruiting Hdac2 to specifically repress IL-6. Nature 2015, 525, 389-393. [CrossRef] [PubMed]

35. Tanaka, S.; Ise, W.; Inoue, T.; Ito, A.; Ono, C.; Shima, Y.; Sakakibara, S.; Nakayama, M.; Fujii, K.; Miura, I.; et al. Tet2 and Tet3 in B cells are required to repress CD86 and prevent autoimmunity. Nat. Immunol. 2020, 21, 950-961. [CrossRef] [PubMed]

36. Xue, S.; Liu, C.; Sun, X.; Li, W.; Zhang, C.; Zhou, X.; Lu, Y.; Xiao, J.; Li, C.; Xu, X.; et al. TET3 Inhibits Type I IFN Production Independent of DNA Demethylation. Cell Rep. 2016, 16, 1096-1105. [CrossRef] [PubMed]

37. Ko, M.; An, J.; Bandukwala, H.S.; Chavez, L.; Aijo, T.; Pastor, W.A.; Segal, M.F.; Li, H.; Koh, K.P.; Lahdesmaki, H.; et al. Modulation of TET2 expression and 5-methylcytosine oxidation by the CXXC domain protein IDAX. Nature 2013, 497, 122-126. [CrossRef]

38. Wang, Y.; Xiao, M.; Chen, X.; Chen, L.; Xu, Y.; Lv, L.; Wang, P.; Yang, H.; Ma, S.; Lin, H.; et al. WT1 recruits TET2 to regulate its target gene expression and suppress leukemia cell proliferation. Mol. Cell. 2015, 57, 662-673. [CrossRef]

39. Chen, L.L.; Lin, H.P.; Zhou, W.J.; He, C.X.; Zhang, Z.Y.; Cheng, Z.L.; Song, J.B.; Liu, P.; Chen, X.Y.; Xia, Y.K.; et al. SNIP1 Recruits TET2 to Regulate c-MYC Target Genes and Cellular DNA Damage Response. Cell Rep. 2018, 25, 1485-1500.e4. [CrossRef]

40. Jin, S.G.; Jiang, Y.; Qiu, R.; Rauch, T.A.; Wang, Y.; Schackert, G.; Krex, D.; Lu, Q.; Pfeifer, G.P. 5-Hydroxymethylcytosine is strongly depleted in human cancers but its levels do not correlate with IDH1 mutations. Cancer Res. 2011, 71, 7360-7365. [CrossRef]

41. Lian, C.; Xu, Y.; Ceol, C.; Wu, F.; Larson, A.; Dresser, K.; Xu, W.; Tan, L.; Hu, Y.; Zhan, Q.; et al. Loss of 5-Hydroxymethylcytosine Is an Epigenetic Hallmark of Melanoma. Cell 2012, 150, 1135-1146. [CrossRef]

42. Takayama, K.; Misawa, A.; Suzuki, T.; Takagi, K.; Hayashizaki, Y.; Fujimura, T.; Homma, Y.; Takahashi, S.; Urano, T.; Inoue, S. TET2 repression by androgen hormone regulates global hydroxymethylation status and prostate cancer progression. Nat. Commun. 2015, 6, 8219. [CrossRef]

43. Puig, I.; Tenbaum, S.P.; Chicote, I.; Arqués, O.; Martínez-Quintanilla, J.; Cuesta-Borrás, E.; Ramírez, L.; Gonzalo, P.; Soto, A.; Aguilar, S.; et al. TET2 controls chemoresistant slow-cycling cancer cell survival and tumor recurrence. J. Clin. Investig. 2018, 128, 3887-3905. [CrossRef]

44. Yang, L.; Yu, S.-J.; Hong, Q.; Yang, Y.; Shao, Z.-M. Reduced Expression of TET1, TET2, TET3 and TDG mRNAs Are Associated with Poor Prognosis of Patients with Early Breast Cancer. PLoS ONE 2015, 10, e0133896. [CrossRef]

45. Wilkins, O.M.; Johnson, K.C.; Houseman, E.A.; King, J.E.; Marsit, C.J.; Christensen, B.C. Genome-wide characterization of cytosine-specific 5-hydroxymethylation in normal breast tissue. Epigenetics 2020, 15, 398-418. [CrossRef]

46. West, A.C.; Johnstone, R.W. New and emerging HDAC inhibitors for cancer treatment. J. Clin. Investig. 2014, 124, 30-39. [CrossRef]

47. Woods, D.M.; Sodre, A.L.; Villagra, A.; Sarnaik, A.; Sotomayor, E.M.; Weber, J. HDAC Inhibition Upregulates PD-1 Ligands in Melanoma and Augments Immunotherapy with PD-1 Blockade. Cancer Immunol. Res. 2015, 3, 1375-1385. [CrossRef]

48. Fukumoto, T.; Fatkhutdinov, N.; Zundell, J.A.; Tcyganov, E.N.; Nacarelli, T.; Karakashev, S.; Wu, S.; Liu, Q.; Gabrilovich, D.I.; Zhang, R. HDAC6 Inhibition Synergizes with Anti-PD-L1 Therapy in ARID1A-Inactivated Ovarian Cancer. Cancer Res. 2019, 79, 5482-5489. [CrossRef]

49. Hegedus, L.; Rittler, D.; Garay, T.; Stockhammer, P.; Kovacs, I.; Dome, B.; Theurer, S.; Hager, T.; Herold, T.; Kalbourtzis, S.; et al. HDAC Inhibition Induces PD-L1 Expression in a Novel Anaplastic Thyroid Cancer Cell Line. Pathol. Oncol. Res. 2020, 26, 2523-2535. [CrossRef] 